\title{
Grid-Forming Control Suitable for Large Power Transmission System Applications
}

\author{
Taoufik Qoria, Xavier Guillaud, Member, IEEE,
}

\begin{abstract}
The inner cascaded structure-based grid-forming control is a typical solution used to impose an AC voltage magnitude across the output filters of the power inverters. Yet, because of the limited inverter's bandwidth resulting from the low-switching frequencies in transmission systems, the interaction (i.e., coupling) between control loops is highly likely making the understanding of the system behavior complex and its simplification unaffordable and may also lead to instabilities. The novelty of this paper consists in proposing a simple open-loop direct voltage control to reduce the number of the inner control regulators, and thereby guaranteeing a decoupling between the inner and outer control layers as well as increasing the system stability margin. This statement is well supported with a smallsignal analysis and progressive order model reduction of the system. The overall concept is validated in a 10-bus grid case while comparing the EMT and Phasor-based simulations. The practical feasibility of the control itself is experimentally proved with different test cases.
\end{abstract}

Index Terms-Grid-forming VSC, AC voltage control, Order Model Reduction, Electromagnetic Transient Simulations, Phasor Simulations.

\section{INTRODUCTION}

$\mathbf{I}$ $\mathrm{N}$ power transmission systems with high penetration rate of inverters-interfaced generation, the way to control the power inverters should be improved or radically changed in order to overcome the limitations of the conventional gridfollowing control [1], [2]. In other words, the way to control power inverters has to be changed from "following the grid" to "supporting the grid". In this context, the grid-forming capability seems to be a suitable alternative for this aim thanks to the potential it brings to the power inverter e.g., voltage source behavior, inherent inertia emulation and the ability to operate in both grid-connected and standalone mode. Several grid-forming variants have been proposed in the literature. These solutions adopt more or less the idea of mimicking the swing equation of a synchronous machine (SM) [3]-[8] to generate the control angle. Regarding the AC voltage magnitude, it is managed by a lower control level (i.e., inner control). One common solution to control the AC voltage magnitude is the cascaded control structure [9]-[11]. This solution is favored for industrial applications because of the simplicity of its implementation and its ability to generate a current reference that serves to protect the inverter against faults. Yet, because of the limited inverter's bandwidth resulting from the low-switching frequencies in transmission systems, the tuning

This work is supported by ELIA Transmission System Operator.

Taoufik Qoria is with Maschinenfabrik Reinhausen, MV-Power Electronics departement EAP, 93059; Regensburg, Germany (t.qoria@reinhausen.com)

Xavier Guillaud is with L2EP, Univ. Lille, Arts et Metiers Institute of Technology, Centrale Lille, Yncrea Hauts-de-France, ULR 2697 Laboratoire d'Electrotechnique et d'Electronique de Puissance, F-59000 Lille, France of the controllers based on this structure is still a challenge making the system stability in a stiff grid condition and the interaction between control layers a major problem [12]. Many studies reported the instability issue of grid-forming VSCs especially in stiff grid conditions, where the stability impact factors were analyzed by the state-space model [13] or the impedance model [14], including the control parameters, the operating point and the grid impedance. Recently, new tuning methods to face this problem have been proposed [9], [10], [15]. However, the explicit control interaction mechanism between control loops leading to this instability was overlooked and few researches broach this research area [16], [17]. In [17], the authors have clearly demonstrated via frequency analysis that the sub-synchronous resonances can be induced in stiff grids, owing to the interaction of inner voltage, current controls and the outer power control loop. In [16], the authors have highlighted the interaction between inner control and outer control loops through a comparison between Phasor and EMT models, in which the voltage and current control parameters play a crucial role. In [11], participation factors have been used to show that the instability of a grid-forming inverter in stiff grid conditions is a consequence of interaction between the current loop and voltage loop. In these prior researches, the control interaction problem is reported through different analysis, however, no solutions have been proposed to guarantee a decoupling between control layers.

The interaction between control loops is not only affecting the system stability, but also making the understanding of system behavior and its simplification for large system' studies unaffordable, which imposes on system operators in the future to use EMT simulations instead of Phasor ones. This affiramation has been claimed in [20], where the authors conclude the noneffectiveness of the phasor approximation method for model reduction in case of high penetration rate of a grid-formingbased power electronics devices. However, it is important to mention that authors in [20] have drawn this conclusion using the cascaded inner control structure in the overall studied power system.

Based on this prior state of the art, the authors of this paper believe that reducing the number of the controllers may be the right option to tackle the problem of interaction and stability. In this context, the direct AC voltage control structure appears an appropriate solution. This concept has already been proposed in [21], [22]. These solutions consist of a mono-voltage control loop based on an integrator controller to regulate the $\mathrm{AC}$ voltage in normal operation. However, in case of faults, the control still need to switch from direct voltage to direct current control, which results in a voltage latch-up and requires a re-tuning of the integrator gain to guarantee a 


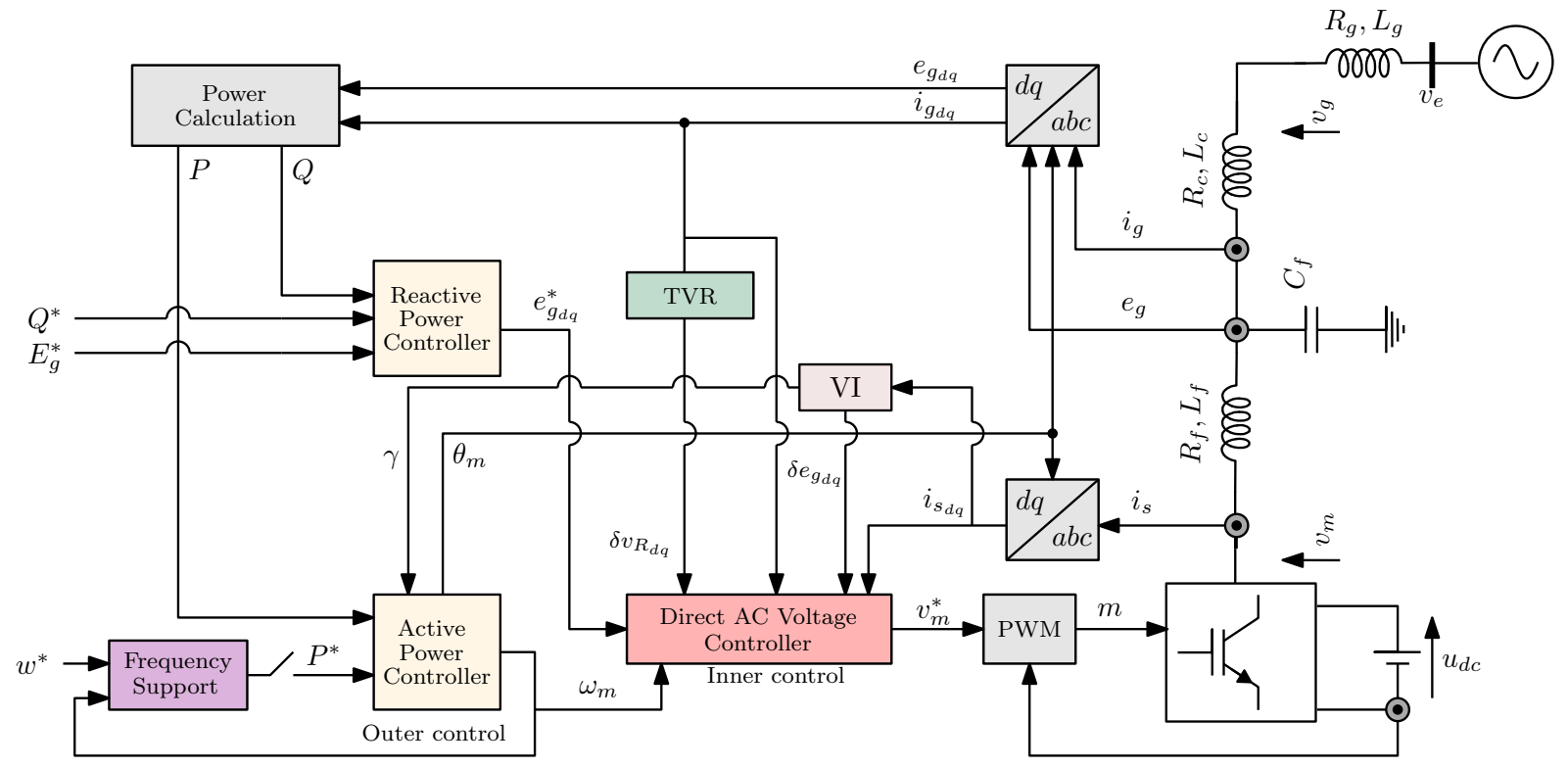

Fig. 1: General configuration of the Current-control free grid-forming VSC

stable switching back to the voltage control mode. From an other side, to the best of our knowledge, no research highlights the benefits of the direct $\mathrm{AC}$ voltage on large power systems in terms of simplicity and equivalence with the Phasor models. This paper looks to fill this gap by proposing the following contributions:

- Recommending extra control functions along this paper to enhance the system operation.

- Proposing a simple open-loop direct AC voltage control with a small-signal stability analysis.

- Assessing the decoupling between different control layers through model order reduction.

- Confirming the effectiveness of RMS simulations compared to EMT ones in a 10-bus grid-case.

The practical feasibility of the control itself is experimentally proved under normal and abnormal conditions. The remaining of this paper is organized as follows. In section II, the gridforming structure is presented and explained. In section III, the direct $\mathrm{AC}$ voltage control is developed and its decoupling with outer controllers is proved and discussed. Section IV validates the proposed control. Finally, section V concludes the paper.

\section{GRID-Forming-BASD Voltage SOURCE INVERTER}

The power model and the control structure of the gridforming VSC are presented in Fig. 1. The VSC is supplied by a perfect $\mathrm{DC}$ bus $u_{d c}$ and connected to the $\mathrm{AC}$ grid through an LC filter and an equivalent transformer impedance. The grid side is modelled by an equivalent Thevenin voltage $v_{e}$ in series with equivalent $R_{g}, L_{g}$. With regard to the control side, the outer control loop consists of the active and reactive power controllers, which generate the voltage angle $\theta_{m}$ and magnitude reference $e_{g_{d q}}^{*}$ with respect to the predefined setpoints and measured power imbalance. The reference voltage vector signal is sent to the voltage regulator, and then to the modulation stage. Each control function illustrated in Fig. 1 is further explained in the following sections.

\section{A. Dynamic equations of the system}

The electrical system consists of an input LC filter $\left(L_{f}, C_{f}\right)$ and a transformer equivalent impedance $L_{c}$ to model the iron losses. It is modeled in the synchronous rotating frame (SRF) defined by the inverter angle $\theta_{m}$. The SRF dynamic equations of the system in s-domain are formulated as follows:

$$
\begin{gathered}
s i_{s_{d}}=\frac{\omega_{b}}{L_{f}}\left(v_{m_{d}}-e_{g_{d}}\right)-\left(\frac{R_{f}}{L_{f}} i_{s_{d}}+\omega_{m} i_{s_{q}}\right) \omega_{b} \\
s i_{s_{q}}=\frac{\omega_{b}}{L_{f}}\left(v_{m_{q}}-e_{g_{q}}\right)-\left(\frac{R_{f}}{L_{f}} i_{s_{q}}-\omega_{m} i_{s_{d}}\right) \omega_{b} \\
s e_{g_{d}}=\frac{\omega_{b}}{C_{f}}\left(i_{s_{d}}-i_{g_{d}}\right)-\omega_{b} \omega_{m} e_{g_{q}} \\
s e_{g_{q}}=\frac{\omega_{b}}{C_{f}}\left(i_{s_{q}}-i_{g_{q}}\right)+\omega_{b} \omega_{m} e_{g_{d}} \\
s i_{g_{d}}=\frac{\omega_{b}}{L_{c}}\left(e_{g_{d}}-v_{g_{d}}\right)-\left(\frac{R_{c}}{L_{c}} i_{g_{d}}+\omega_{m} i_{g_{q}}\right) \omega_{b} \\
s i_{g_{q}}=\frac{\omega_{b}}{L_{c}}\left(e_{g_{q}}-v_{g_{q}}\right)-\left(\frac{R_{c}}{L_{c}} i_{g_{q}}-\omega_{m} i_{g_{d}}\right) \omega_{b}
\end{gathered}
$$

The active and reactive power formulas in SRF are given by:

$$
\begin{gathered}
P=e_{g d} i_{g d}+e_{g q} i_{g q} \\
Q=-e_{g d} i_{g q}+e_{g q} i_{g d}
\end{gathered}
$$

The system state variables are the VSC output current $i_{s}$ flowing through the filter $L_{f}$, the AC voltage $e_{g}$ across the capacitor filter $C_{f}$ and the grid current $i_{g}$ flowing through the transformer impedance $L_{c}$, respectively. The modulated and the grid voltages are denoted by $v_{m}$ and $v_{g} . \omega_{b}$ represents the system base frequency and $\omega_{m}$ is the inverter frequency in per-unit. 


\section{B. Power Control}

In this paper, the outer active power controller is based on the PLL-free PI-controller [8]:

$$
\begin{gathered}
\omega_{m}=\frac{1}{2 H s}\left(\gamma P^{*}-P\right)-k_{p} P \\
\theta_{m}=\omega_{m} \omega_{b} / s
\end{gathered}
$$

with $H, k_{p}$ and $P^{*}$ denote the inertia constant, the damping factor and the active power setpoint, respectively. $\theta_{m}$ denotes the time-domain angle. $\gamma$ is an adaptive gain that aids the gridforming to stably resynchronize after large disturbances [25]. It is determined by the virtual impedance control when the current exceeds its rated value (see subsection D).

In addition to the ability of the PI-controller to provide an inertial response, it takes the advantage of guaranteeing a decoupling between the active power regulation and the frequency support function exactly as a VSM [8], but without a need to a dedicated PLL, which makes the power control much simpler.

The frequency support block is a classical frequency droop control:

$$
P^{*}=\frac{1}{R}\left(\omega^{*}-\omega_{m}\right)
$$

$\mathrm{R}$ denotes the droop gain.

Regarding the reactive power outer controller, it is based on the conventional reactive droop function:

$$
Q^{*}-Q\left(\frac{1}{1+T_{Q} s}\right)=\frac{1}{n_{q}}\left(e_{g_{d}}^{*}-E_{g}^{*}\right)
$$

with $Q^{*}, E_{g}^{*}, n_{q}$ and $T_{Q}$ denote the reactive power reference setpoint, the voltage setpoint, the reactive droop gain and the reactive power time constant, respectively.

\section{Transient Virtual Resistor (TVR)}

During transients, maintaining a constant voltage would induce synchronous oscillations due to the undamped line dynamics $(X \gg R)$ [12], [21], especially in case of stiff grid conditions. These oscillations may result in unstable system operation in case of fast active power dynamics $\left(T_{R}^{5 \%} \leq\right.$ 100ms) [2]. To avoid the consequent instability, a TVR should be adopted in the control to damp the grid current dynamics and thereby, ensures a frequency decoupling between the grid current dynamics and the power control dynamics. The TVR expression is given in the following line:

$$
\delta v_{R_{d q}}=R_{V}\left(\frac{s}{\omega_{f}+s}\right) i_{g_{d q}}
$$

$R_{v}$ is the virtual resistor. $\omega_{f}$ is the cut-off frequency of the high-pass filter.

\section{Virtual impedance for current limitation (VI)}

The VI principle consists in increasing the output impedance during the overcurrent period in order to reduce the inverter voltage magnitude and accordingly limiting the output current. This solution has been used in this paper because of the absence of a current regulation loop in the proposed control, and also because of its ability to improve the transient stability of the system.

As illustrated in Fig. 2, the VI operation principle is divided into two main phases:

- Overcurrent detection and VI calculation (activates only when the current exceeds its nominal value $I_{n}=1 \mathrm{p} . \mathrm{u}$ ).

- AC voltage drop calculation.

The expressions of $X_{V I}$, and $R_{V} I$ are given in (14) and (15).

$$
\begin{gathered}
X_{V I}=\left\{\begin{array}{lll}
k_{p_{R_{V I}}} \delta I \sigma_{X / R} & \text { IF } & \delta I>0 \\
0 & \text { IF } & \delta I \leq 0
\end{array}\right. \\
R_{V I}=X_{V I} \sigma_{X / R}^{-1}
\end{gathered}
$$

where $\delta I=I_{s}-I_{n} . k_{p_{R_{V I}}}$ and $\sigma_{X / R}$ are defined respectively as the virtual impedance proportional gain and the virtual impedance ratio. The parameter $k_{p_{R_{V I}}}$ is tuned to limit the current magnitude to a suitable level $I_{\max }$ in steady state.

When the virtual impedance is activated, the new AC voltage references are given by (16) and (17):

$$
\begin{gathered}
e_{g_{d}}^{*}=e_{g_{d}}^{*}-\delta e_{g_{V I_{d}}} \\
e_{g_{d}}^{*}=-\delta e_{g_{V I_{q}}}
\end{gathered}
$$

with:

$$
\begin{aligned}
\delta e_{g_{V I}} & =\left(R_{V I}+K_{D}\right) i_{s_{d}}-X_{V I} i_{s_{q}} \\
\delta e_{g_{V I}} & =\left(R_{V I}+K_{D}\right) i_{s_{q}}+X_{V I} i_{s_{d}}
\end{aligned}
$$

\section{E. Post-fault Re-synchronization algorithm}

In case of large disturbances, the resulting power mismatch during faults may lead to a loss of synchronism. To face this issue, an adaptive gain $\gamma$ given in (20) is implemented in the power control loop (see (9)) in order to adapt the active power reference with respect to the voltage generated by the VI. If the current does not reach its maximum allowable value $I_{\max }$, the gain $\gamma$ is equal to 1 . While, when a fault occurs and the current is above its rated value, the factor $\gamma$ is modified with respect to the VI voltage leading a decrease of the power reference, and thereby to a larger angle stability margin. More information on this gain are found in [25].

$$
\gamma=\sqrt{\left(1-\delta e_{g d}\right)^{2}+\delta e_{g q}^{2}}
$$

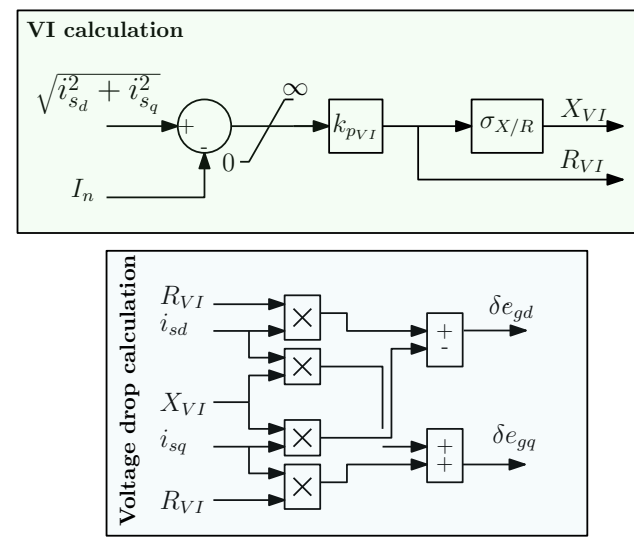

Fig. 2: VI structure 


\section{Direct AC Voltage Control \& ORder Model REDUCTION}

\section{A. Principle of the Direct AC Voltage Control}

The aim of the inner control is to set a specified voltage magnitude across the capacitor $C_{f}$. To achieve this objective, the modulated AC voltage reference $v_{m_{d q}}^{*}$ is generated from the voltage setpoint $e_{g_{d q}}^{*}$ and the inverse of the quasi-stationary model of the filter $R_{f}, L_{f}$ :

$$
\begin{aligned}
& v_{m_{d}}^{*}=e_{g_{d}}^{*}+R_{f} i_{d}-L_{f} \omega_{m} i_{s_{q}} \\
& v_{m_{q}}^{*}=e_{g_{q}}^{*}+R_{f} i_{q}+L_{f} \omega_{m} i_{s_{d}}
\end{aligned}
$$

$R_{f}, L_{f}$ are known transformer parameters.

By neglecting the $\mathrm{PWM} /$ measurement delays and replacing (21-22) in (1-2), respectively, the AC voltage $e_{g}$ will be equal to its voltage reference in steady-state:

$$
e_{g_{d q}} \approx e_{g_{d q}}^{*}
$$

The result in (23) is achievable assuming a good estimation of the filter parameters $\left(R_{f}, L_{f}\right)$. If the estimation is not accurate, the resulting slight voltage deviation will be compensated by the reactive power control or the secondary voltage control not presented in this work.

To avoid particular resonance linked to the LCL filter, an active damping control has to be implemented:

$$
\begin{aligned}
& v_{m_{d}}^{*}=e_{g_{d}}^{*}+R_{f} i_{d}-L_{f} \omega_{m} i_{s_{q}}-R_{L C L_{D}}\left(i_{s_{d}}-i_{g_{d}}\right) \\
& v_{m_{q}}^{*}=e_{g_{q}}^{*}+R_{f} i_{q}+L_{f} \omega_{m} i_{s_{d}}-R_{L C L_{D}}\left(i_{s_{q}}-i_{g_{d q}}\right)
\end{aligned}
$$

The active damping control in (24) and (25) acts as a physical resistor in series with the filter capacitor $C_{f}$ (Fig. 3). The active damping may induce a considerable $\mathrm{AC}$ voltage drop with respect to the amount of the exchanged power. Therefore, a high-pass filter is implemented so that the damping resistor only reacts to transients.

$$
\begin{aligned}
& v_{m_{d}}^{*}=e_{g_{d}}^{*}+R_{f} i_{d}-L_{f} \omega_{m} i_{s_{q}}-\frac{R_{L C L_{D}} s}{\omega_{R}+s}\left(i_{s_{d}}-i_{g_{d}}\right) \\
& v_{m_{q}}^{*}=e_{g_{q}}^{*}+R_{f} i_{q}+L_{f} \omega_{m} i_{s_{d}}-\frac{R_{L C L_{D}} s}{\omega_{R}+s}\left(i_{s_{q}}-i_{g_{q}}\right)
\end{aligned}
$$

The inner control structure derived from (26-27) is depicted in Fig. 4.

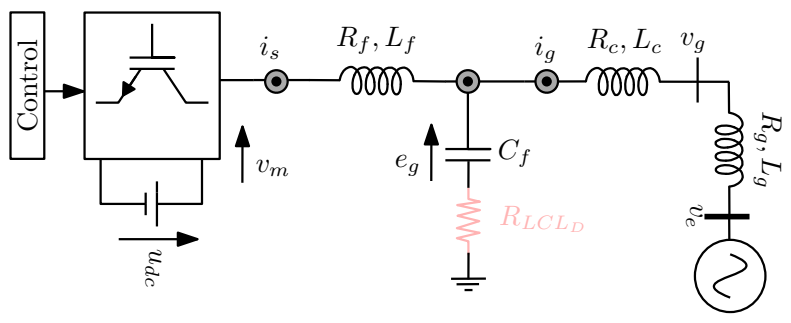

Fig. 3: Emulation of the LCL damping resistor

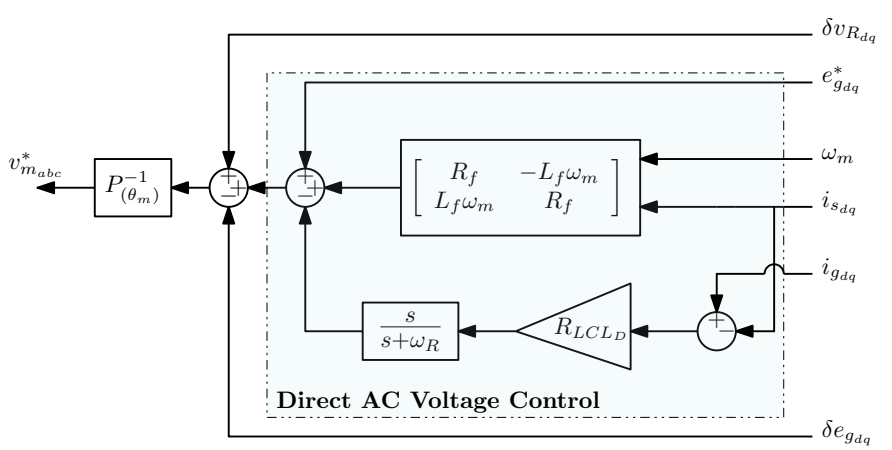

Fig. 4: Direct AC voltage control scheme

TABLE I: System and control parameters

\begin{tabular}{|c|c||c|c|}
\hline$P_{n}$ & $1000 \mathrm{MW}$ & $f_{n}$ & $50 \mathrm{~Hz}$ \\
\hline $\cos \phi$ & 0.95 & $U_{e}$ & $320 \mathrm{kV}$ \\
\hline $\mathrm{SCR}$ & 10 & $e_{g_{d}}^{*}$ & $1 \mathrm{p.u}$ \\
\hline$L_{c}$ & $0.15 \mathrm{p.u}$ & $e_{g_{q}}^{*}$ & 0 \\
\hline$R_{c}$ & $0.005 \mathrm{p} . \mathrm{u}$ & $\omega_{f}$ & $60 \mathrm{rad} / \mathrm{s}$ \\
\hline$C_{f}$ & $0.066 \mathrm{p} . \mathrm{u}$ & $k_{p}$ & $0.015 \mathrm{p} . \mathrm{u}$ \\
\hline$R_{v}$ & $0.09 \mathrm{p} . \mathrm{u}$ & $L_{f}$ & $0.15 \mathrm{p} . \mathrm{u}$ \\
\hline$R_{f}$ & $0.005 \mathrm{p} . \mathrm{u}$ & $H$ & $5 \mathrm{~s}$ \\
\hline$n_{q}$ & 0 & $R$ & $5 \%$ \\
\hline$u_{d c}$ & $640 \mathrm{kV}$ & $I_{m a x}$ & $1.2 \mathrm{p} . \mathrm{u}$ \\
\hline$I_{n}$ & $1 \mathrm{p} . \mathrm{u}$ & $\sigma_{X / R}$ & 5 \\
\hline$k_{p R_{V I}}$ & 0.67 & & \\
\hline
\end{tabular}

\section{B. Tuning and Small-Signal Stability Analysis of The System Based on The Direct AC voltage Control}

In order to assess the system stability and dynamics with respect to the control parameters, the model of the gridforming inverter depicted in Fig. 1 is linearized around an operating point $\left(P_{0}=1\right.$ p.u):

$$
\left\{\begin{array}{l}
\Delta \dot{\mathbf{x}}=\mathbf{A} \Delta \mathbf{x}+\mathbf{B} \Delta \mathbf{u} \\
\Delta \mathbf{y}=\mathbf{C} \Delta \mathbf{x}+\mathbf{D} \Delta \mathbf{u}
\end{array}\right.
$$

with:

$$
\begin{gathered}
x=\left[\begin{array}{ccccccc}
e_{g_{d}} & e_{g_{q}} & i_{s_{d}} & i_{s_{q}} & i_{g_{d}} & i_{g_{q}} \delta \omega_{m} & \delta_{m} \cdots \\
\delta e_{g} & \zeta_{L C L D_{d}} & \zeta_{L C L D_{q}} & \zeta_{T V R_{d}} & \zeta_{T V R_{q}}
\end{array}\right]^{T} \\
u=\left[\begin{array}{llll}
E_{g}^{*} & V_{e} & P^{*}
\end{array}\right]^{T}
\end{gathered}
$$

- $e_{g_{d q}}, i_{g_{d q}}, i_{s_{d q}}$ are the LCL filter state variables.

- $\delta \omega_{m}, \delta_{m}$ are the active power controller state variables.

- $\delta e_{g}$ is the reactive power filter state variable.

- $\zeta_{T V R_{d}}, \zeta_{T V R_{q}}$ are the state variables of TVR filters.

- $\zeta_{L C L D_{d}}, \zeta_{L C L D_{q}}$ are the LCL active damping control state variables.

System and control parameters are presented in Table I.

To check the impact of the active damping control on the poles location, $R_{L C L D}$ is varied from 0 to 1 p.u for two values of $\omega_{R}$ as shown in Fig. 5. One can notice from the eigenvalues evolution $\left(\lambda_{1-2}, \lambda_{3-4}\right)$ that without additional transient active damping, the system will be unstable. The parameter $R_{L C L_{D}}>0.1$ p.u enhances the stability and damps the oscillatory modes. The parameter $\omega_{R}$ do not have any noticeable impact on stability, however, from an experimental point of view, the choice of a small cut-off frequency allows 
avoiding the amplification of the measurements noise. Thus, $\omega_{R}$ and $R_{L C L_{D}}$ are set in the following to $10 \mathrm{rad} / \mathrm{s}$ and 0.7 p.u, respectively. The chosen value of $R_{L C L_{D}}$ is sufficient to guarantee a frequency decoupling between the inner and outer loops. This statement is confirmed in the subsection III.C. Based on the chosen parameters, the system eigenvalues are listed in Table II. To illustrate the dynamic behavior of the system based on the proposed strategy, an active power setpoint change of $P^{*}=1 \mathrm{p} . \mathrm{u}$ is applied to the system at $t=1 s$. The simulation results are gathered in Fig. 6. They prove the stability of the system and the ability of the direct $\mathrm{AC}$ voltage control to ensure $\mathrm{AC}$ voltage tracking in steady state after disturbance.

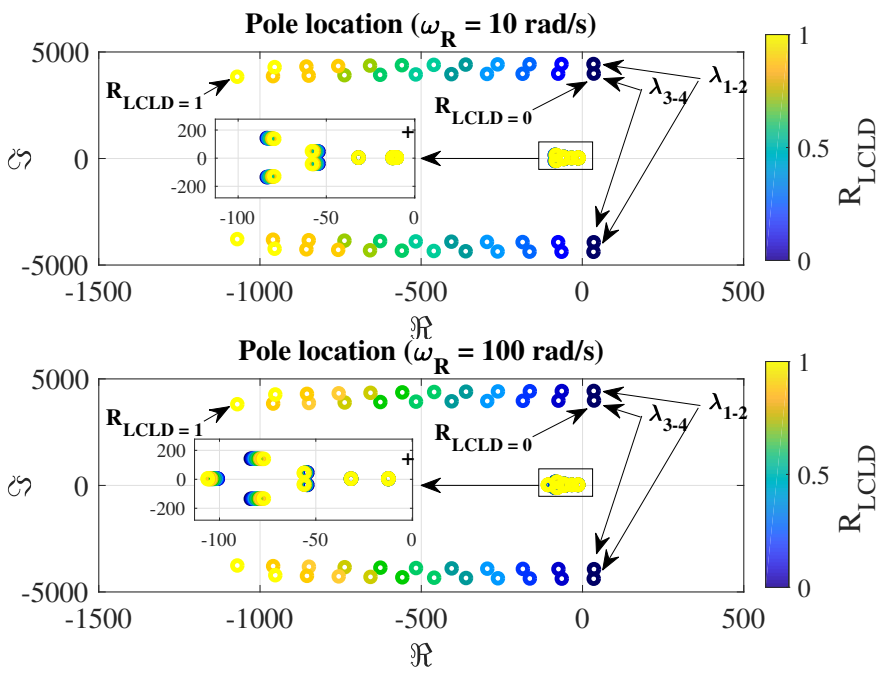

Fig. 5: Poles Location with respect to $R_{L C L D}$ and $\omega_{R}$

TABLE II: System Eigenvalues

\begin{tabular}{|c|c||c|c|}
\hline$\lambda_{1-2}$ & $-686.4 \pm 3735.4 \mathrm{i}$ & $\lambda_{8-9}$ & $-60.9 \pm 5.47 \mathrm{i}$ \\
\hline$\lambda_{3-4}$ & $-766.4 \pm 3346 \mathrm{i}$ & $\lambda_{10-11}$ & $-5.17 \pm 6.19 \mathrm{i}$ \\
\hline$\lambda_{5-6}$ & $-32.31 \pm 233.65 \mathrm{i}$ & $\lambda_{12-13}$ & $-9.97 \pm 0.46 \mathrm{i}$ \\
\hline$\lambda_{7}$ & $-31.42 \pm 0 \mathrm{i}$ & & \\
\hline
\end{tabular}
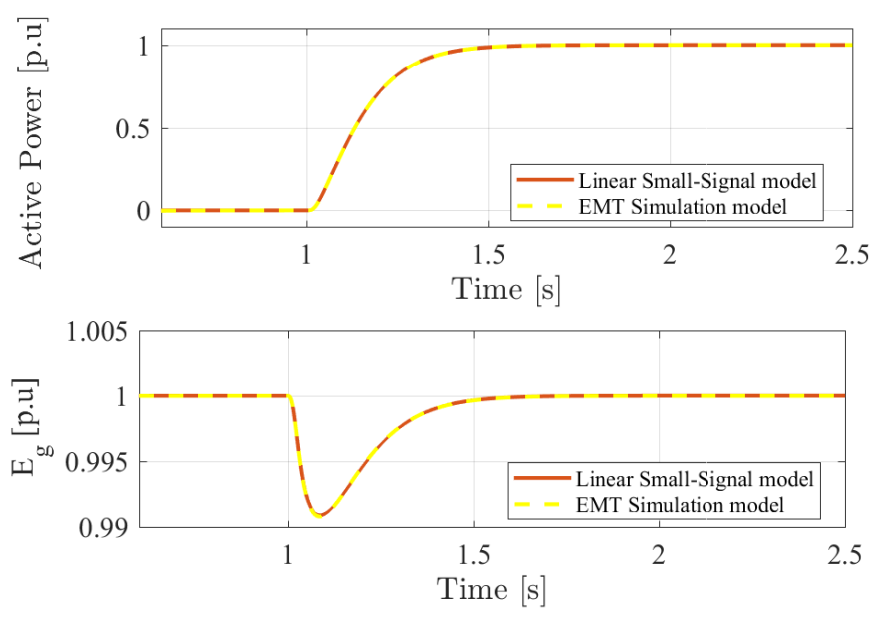

Fig. 6: System dynamics in response to the active power change

\section{Order Model reduction and control layers decoupling}

In large power system, model reduction is of a high interest. It is used to ease the analysis system and to reduce the time computations. The Phasor approximation is one of the model order reduction methods often used for power system analysis. It consists of discarding the fastest dynamics of some state variables $x_{i}$ by freezing their derivative $\dot{x}_{i}=0$ and maintaining the slowest ones that define the dominant response of the system. This method is valid provided that the discarded states do not impact the dominant response of the system.

This section aims to demonstrate the decoupling between different system and control modes. A first step consists in finding the link between the fastest eigenvalues $\lambda_{i}$ in Table II and the state variables $x_{i}$. The aim is to define clearly the state variables to discard and those to keep. The link between eigenvalues $\lambda_{i}$ the state variables $x_{i}$ is usually given by the participation factors. The participation of the state variables $x_{i}$ in each eigenvalue $\lambda_{i}$ is summarized in Table III. In Table III, the eigenvalues are ranked from the fastest to the slowest ones. One can notice that the state variables $e_{g_{d q}}, i_{g_{d q}}, i_{s_{d q}}, \zeta_{T V R_{d q}}$ ,$\zeta_{L C L D_{d q}}$ and $\delta e_{g}$ are participating in the fastest eigenvalues $\lambda_{1-2}, \lambda_{3-4}, \lambda_{5-6}, \lambda_{8-9}, \lambda_{7}$ and $\lambda_{12-13}$. Therefore, in the case they are neglected, they do not impact the dominant modes $\lambda_{10-11}$ linked to the active power dynamics $\delta_{m}$ and $\delta \omega_{m}$. By doing so, the system order can be reduced from $13^{\text {th }}$ to $2^{\text {nd }}$ order (Phasor model), where only the active power dynamics are preserved.To show the system behavior with different levels of reduction, two test cases are performed:

- Active power setpoint change of $P^{*}=1$ p.u.

- Grid voltage phase jump of $-10 \mathrm{deg}$.

The results are gathered in Fig.7. Fig.7.a shows that the discarded modes at each stage $\left(13^{\text {th }}, 7^{\text {th }}, 2^{\text {nd }}\right)$ do not impact the location of the dominant modes (e.g., $7^{\text {th }}$ order neglecting the grid currents, the inverter currents and the inverter output capacitor, the $2^{\text {nd }}$ order keeping only the active power controller states). This result is also highlighted in Fig.7.b and Fig.7.c through time-domain simulations, where the active power response is nearly the same for both test cases. Based on this study, it is concluded that the inner loops based on the direct voltage control do not interact with the outer power control loop, and thereby, the system dynamics in the case of active power change and phase jump can be simply studied using the simplified linear quasi-static model in Fig. 8. To consolidate the contribution of the direct control structure compared to the conventional cascaded structure. Simulation results from [16] are recalled in this paper in Fig. 9. It shows a wide difference between the Phasor and EMT models.

TABLE III: Participation Factors

\begin{tabular}{|c||c|}
\hline Eigenvalues $\lambda_{i}$ & Participating state variables $x_{i}$ \\
\hline \hline$\lambda_{1-2}$ & $e_{g_{d q}}, i_{g_{d q}}, i_{s_{d q}}$ \\
\hline$\lambda_{3-4}$ & $e_{g_{d q}}, i_{g_{d q}}, i_{s_{d q}}$ \\
\hline$\lambda_{8-9}$ & $\zeta_{T V R_{d q}}$ \\
\hline$\lambda_{5-6}$ & $i_{g_{d q}}, i_{s_{d q}}$ \\
\hline$\lambda_{7}$ & $\delta e_{g}$ \\
\hline$\lambda_{12-13}$ & $\zeta_{L C L D_{d q}}$ \\
\hline$\lambda_{10-11}$ & $\delta_{m}, \delta \omega_{m}$ \\
\hline
\end{tabular}




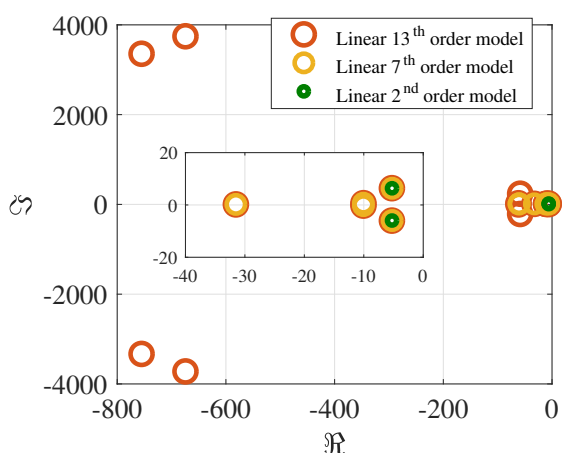

(a)

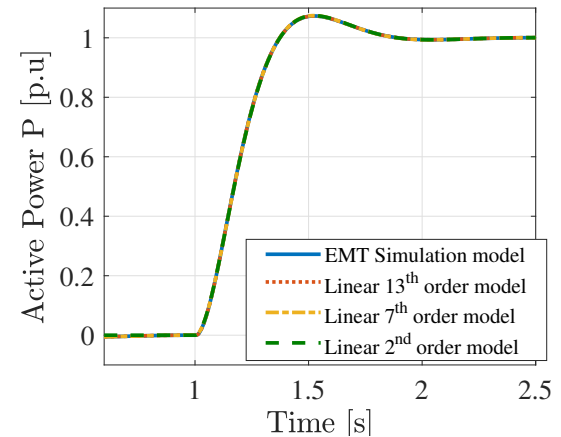

(b)

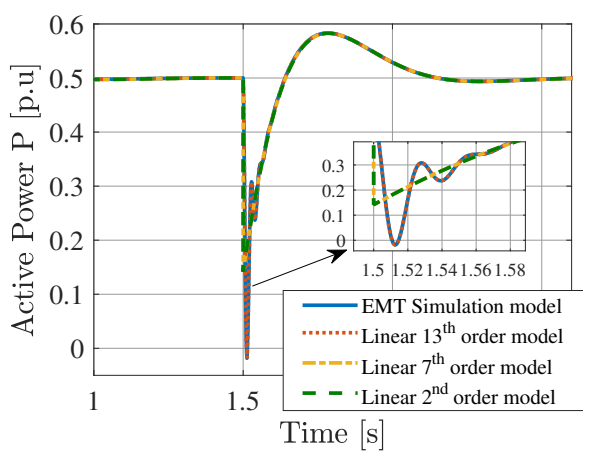

(c)

Fig. 7: System performances with order reduction. (a) System modes. (b) Active power change. (c) $-10^{\circ}$ phase jump

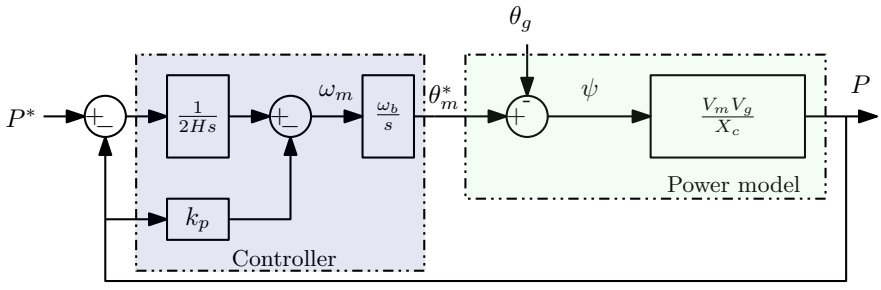

Fig. 8: Phasor model [8]

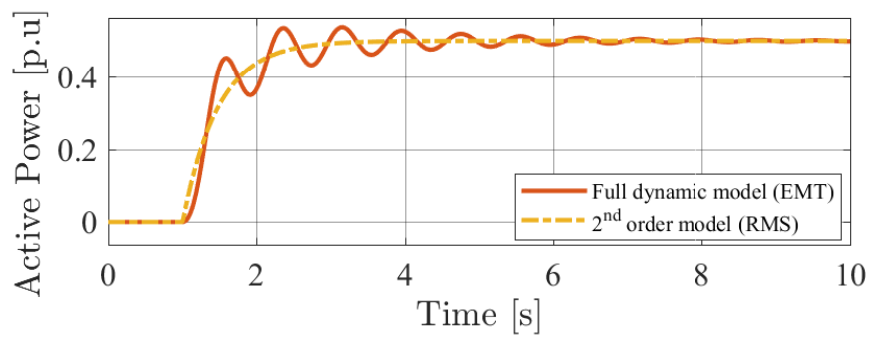

Fig. 9: Comparison between EMT-based model with a cascaded controller structure and the Phasor model following an active power change [16]

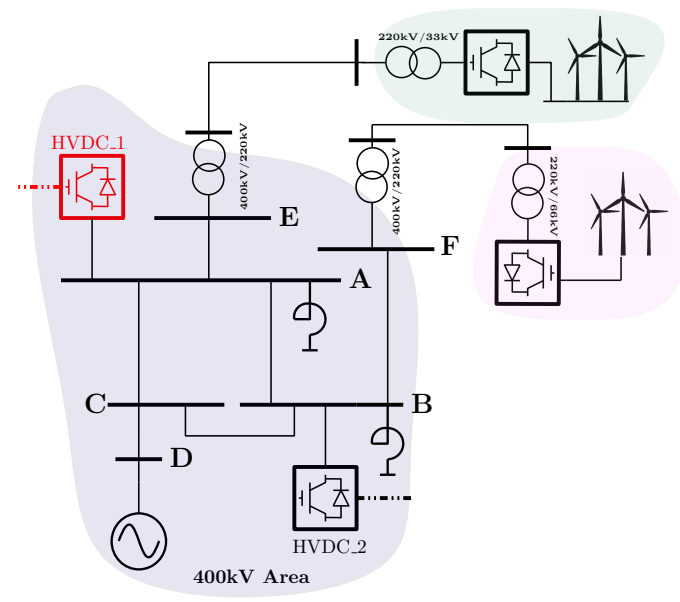

Fig. 10: 10-bus grid case

\section{10-bus grid case study}

In this subsection, the grid-forming converter depicted by HVDC-1 in Fig. 10 is connected to a more realistic grid configuration instead of a perfect voltage source. The latter consists of two Wind Parks $(33 \mathrm{kV}$ and $66 \mathrm{kV})$ interfaced to the $400 \mathrm{kV}$ power system through grid-following basedinverters and two step-up transformers, 1 GVA HVDC-2 gridfollowing-based inverter and an equivalent 33 GVA AC grid driven by an equivalent model of synchronous generator [5]. In order to excite the system modes and especially those of the grid-forming inverter, two extreme test cases are applied for the system: 1) a tripping of the corridor between bus A and bus C. 2) $250 \mathrm{~ms}$ bolted-fault at the bus B. The results are given in Fig. 11 and Fig. 12, respectively, where both EMTBased EMTP-RV and RMS-based Ramses [26] simulations are compared.

The tripping of the corridor between bus $\mathrm{A}$ and $\mathrm{C}$ engenders a significant phase jump and a voltage variation due to the amount of active and reactive power transported at this level, which results in $1800 \mathrm{MW}$ active power variation in the transient at bus $\mathrm{A}$ and 250MVAR reactive power injection at both A and B buses to support the AC voltage (see Fig. 11). Despite the severity of the event, the system remains stable and the overall behavior of the system simulated by EMT program is quite close to the one simulated by RAMSESPhasor program. This conclusion is reinforced through the three-phase short-circuit test case illustrated in Fig. 12. This test yields an overcurrent, which is accurately limited by the virtual impedance to 1.2 p.u as required, and once again, the EMT simulations match the Phasor ones.

\section{EXPERIMENTAL VALIDATION}

The aim of this section is to validate experimentally the system operation based on the proposed control. The experimental bench is illustrated in Fig. 13. The 2-Level VSC (1) is supplied by an ideal $600 \mathrm{~V}$ DC voltage source (4) and connected to a high bandwidth AC amplifier (5) through an LCL filter (3). The amplifier is used to emulate the AC system (i.e.; $300 \mathrm{~V}$ phph) as well as to generate the events discussed in this paper (i.e.; active power change, $100 \%$ voltage sag emulating a 3phase bolted fault and a phase shift). The 2-Level VSC is controlled with a dSPACE dS1005 (2) with a 40us time step. The switching frequency of the inverter is $f_{s w}=10 \mathrm{kHz}$. System parameters are listed in Table IV. 

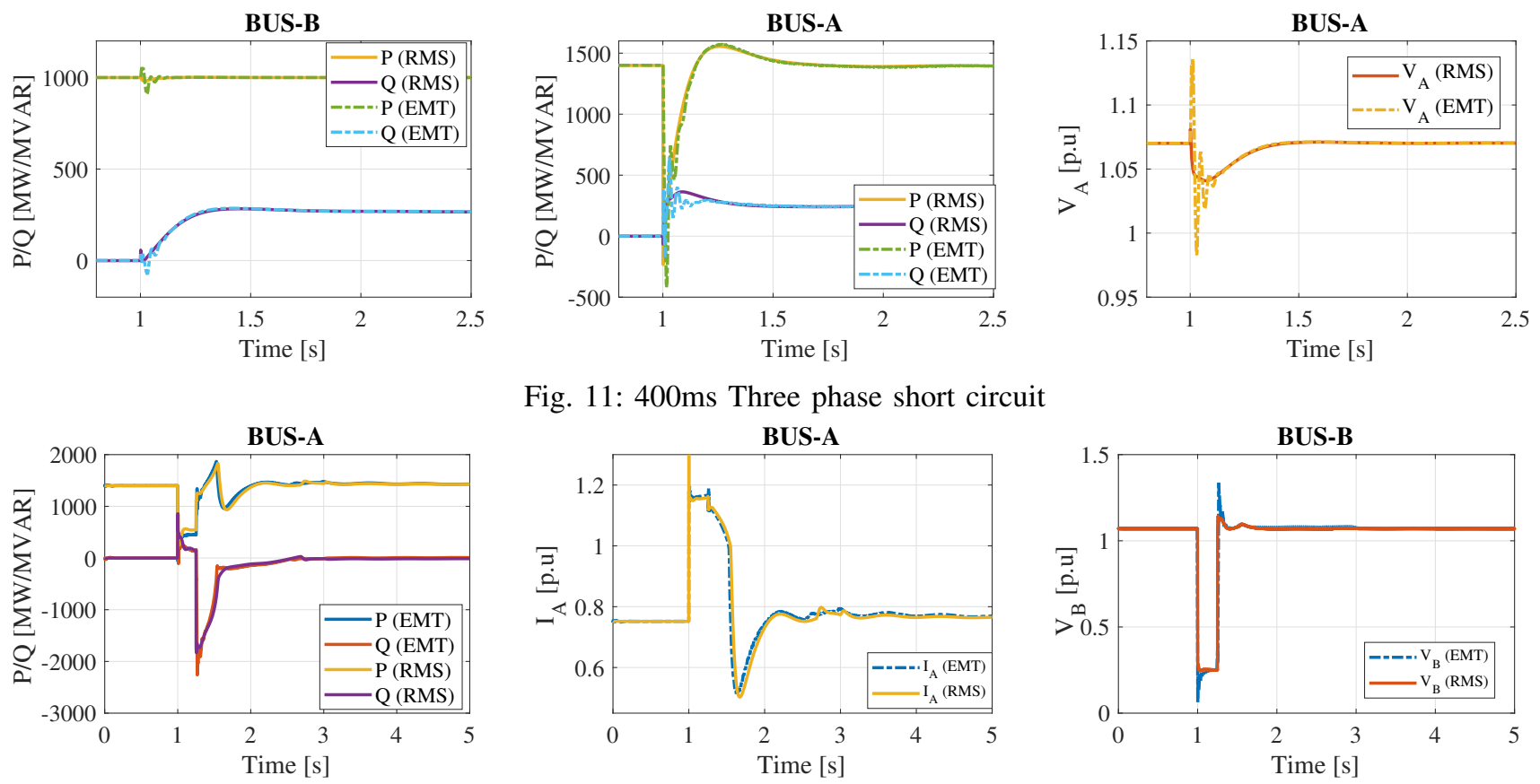

Fig. 11: 400ms Three phase short circuit
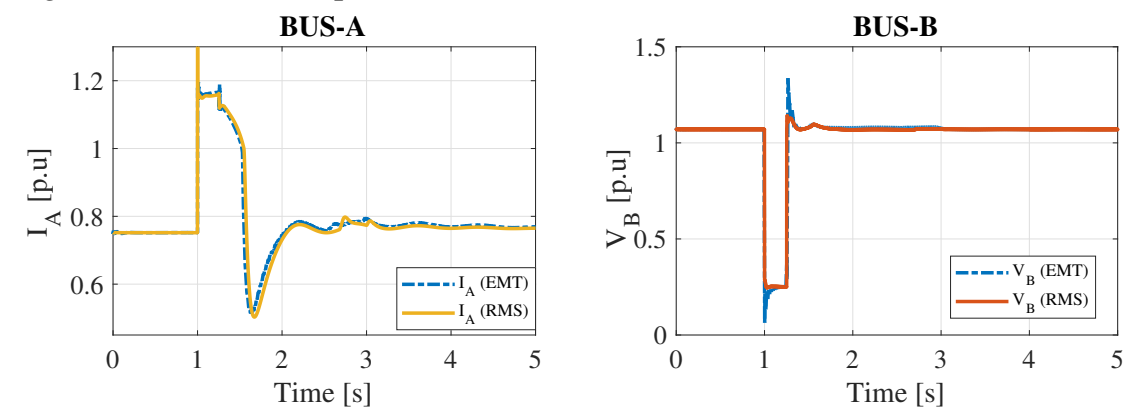

Fig. 12: 400ms Three phase short circuit

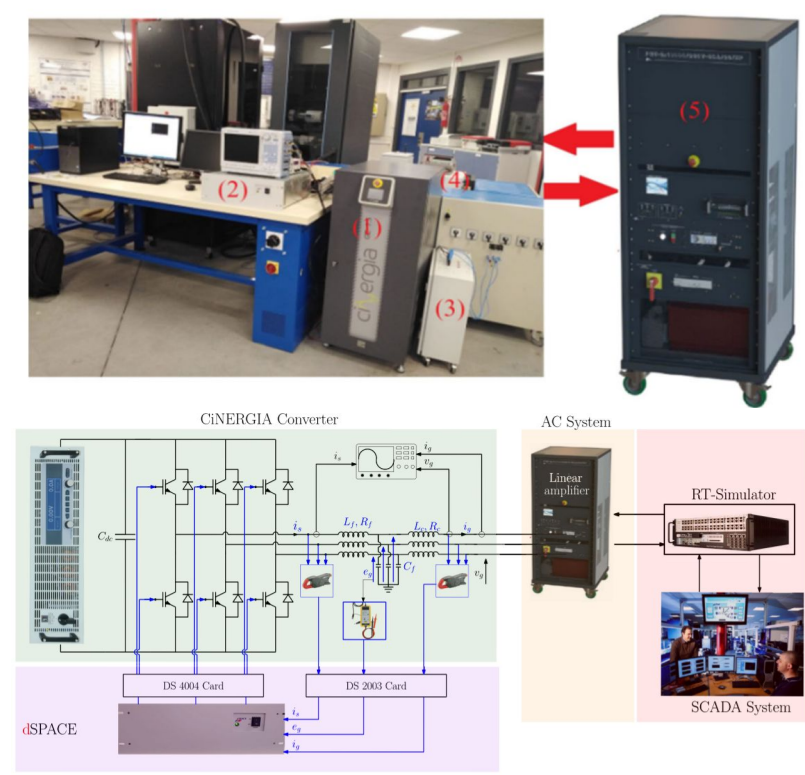

Fig. 13: Mockup presentation \& Functional scheme

The electrical quantities displayed in the experimentation are respectively the grid voltages $v_{g}$, the grid current $i_{g}$ (plotted in the oscilloscope), the active power $p_{a c}$ and the inverter output voltage $e_{g}$ (plotted in dSPACE). Two test cases are performed: a 0.4 active power change and $100 \%$ grid voltage sag.

TABLE IV: System Parameters

\begin{tabular}{|c|c||c|c|}
\hline$S_{n}$ & $5.62 \mathrm{kVA}$ & $I_{n}$ & $10 \mathrm{~A}$ \\
\hline$L_{f}$ & $10.9 \mathrm{mH}$ & $L_{c}$ & $10.9 \mathrm{mH}$ \\
\hline$f_{n}$ & $50 \mathrm{~Hz}$ & $C_{f}$ & $9.2 u f$ \\
\hline
\end{tabular}

\section{A. Active power setpoint change}

The power reference is first set to $P^{*}=0.1$ p.u. At $\mathrm{t}$ $=3.95 \mathrm{~s}, \Delta P^{*}=0.4$ p.u active power change is applied as illustrated in Fig. 14. One can first notice that the system is stable. Additionally, the AC voltage drop resulted from the active power change is compensated thanks to the inverted impedance $\left(L_{f}, R_{f}\right)$, which yields a negligible $\mathrm{AC}$ voltage error in steady state $(\epsilon=1.6 \%)$.

\section{B. $100 \%$ Voltage sag}

Initially, $P^{*}=0.5$ p.u. Subsequently, a $400 \mathrm{~ms}$ voltage sag is applied as shown in Fig. 15. When the fault occurs, the AC voltage drop induces an increase in the VSC output current, which is limited beyond its maximum allowable current magnitude, 1.2 p.u (12 A). Once the fault is cleared, the system stably recovers its equilibrium point.

\section{CONClusion}

This paper proposes a grid-forming strategy suitable for power transmission system application. It has been highlighted in this paper that the proposed control is very simple and guarantees a well decoupling between different control layers. It has also been demonstrated that the dominant modes of the system associated with the outer power control dynamics are preserved, which allows neglecting the inner dynamics. This satisfies the prerequisite of using Phasor approximation for dynamic studies in large power systems. The feasibility of the proposed control has been proved through experimentation in two faulty cases.

\section{ACKNOWLEDGMENTS}

The Authors gratefully acknowledge the contributions of: Thierry Van Cutsem, Bertrand Bastin, Antonin Colot from 

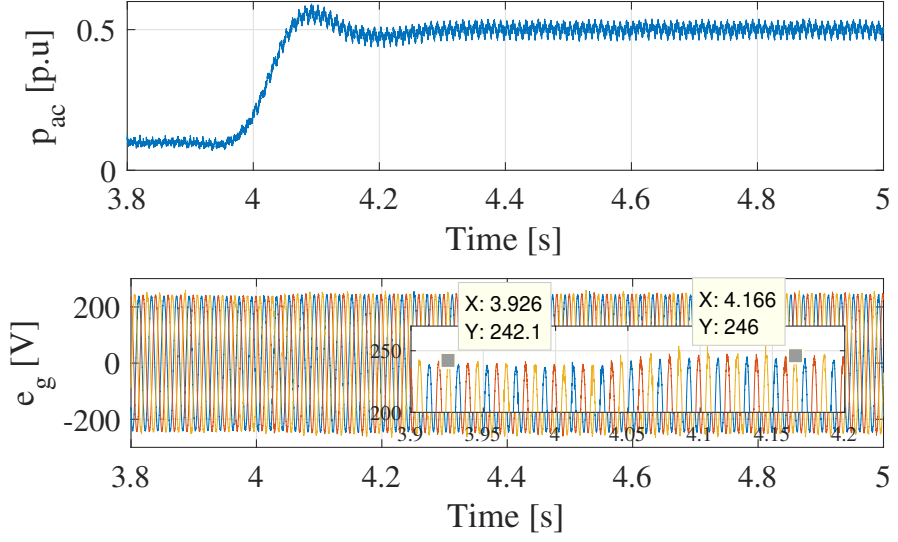

Fig. 14: Active Power change

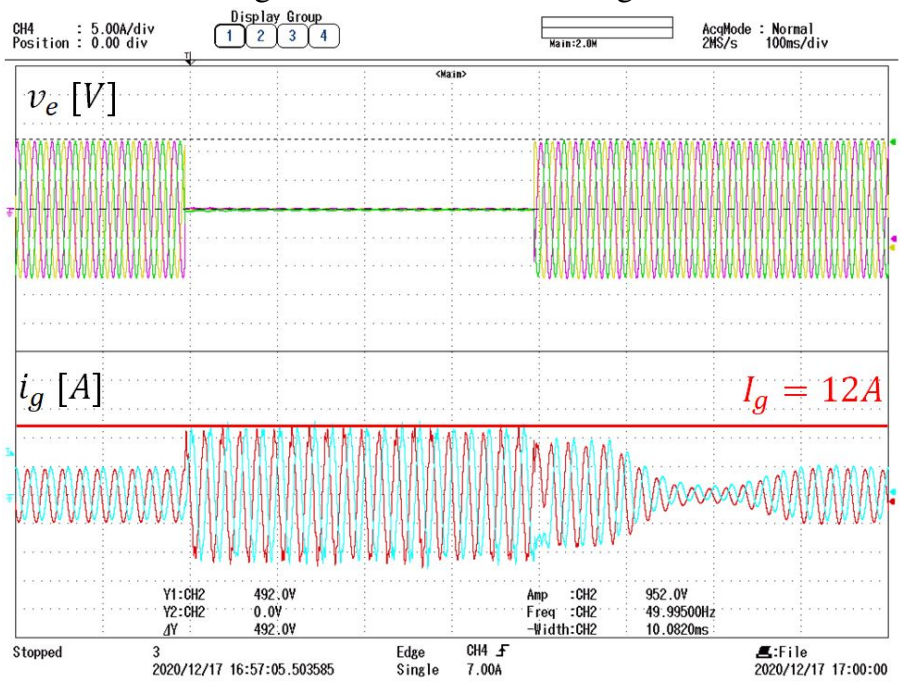

Fig. 15: 400ms Three phase short circuit

University of Liege, Dept. Electrical Engineering and Comp. Sc. Belgium and Dr. Riad Kadri for ENSAM Lille France.

\section{REFERENCES}

[1] "High Penetration of Power Electronic Interfaced Power Sources (HPoPEIPS)," ENTSO-E, Technical, Mar. 2017. [Online]. Available: https://consultations.entsoe.eu/system-development/entso-e-connectioncodes-implementation-guidance-d-3

[2] T. QORIA, "Grid-forming control to achieve a $100 \%$ power electronics interfaced power transmission systems," ENSAM, Paris, 2020.

[3] S. D'Arco and J. A. Suul, "Equivalence of Virtual Synchronous Machines and Frequency-Droops for Converter-Based MicroGrids," IEEE Trans. Smart Grid, vol. 5, no. 1, pp. 394-395, Jan. 2014, doi: 10.1109/TSG.2013.2288000.

[4] J. Liu, Y. Miura, and T. Ise, "Comparison of Dynamic Characteristics Between Virtual Synchronous Generator and Droop Control in InverterBased Distributed Generators," IEEE Trans. Power Electron., vol. 31, no. 5, pp. 3600-3611, May 2016, doi: 10.1109/TPEL.2015.2465852.

[5] T. QORIA, F. GRUSON, F. COLAS, G. Denis, T. PREVOST, and G. Xavier, "Inertia effect and load sharing capability of grid forming converters connected to a transmission grid," The 15th IET international conference on AC and DC Power Transmission, UK, p. 6, Jan. 2019.

[6] M. Ashabani, F. D. Freijedo, S. Golestan, and J. M. Guerrero, "Inducverters: PLL-Less Converters With Auto-Synchronization and Emulated Inertia Capability," IEEE Trans. Smart Grid, vol. 7, no. 3, pp. 1660-1674, May 2016, doi: 10.1109/TSG.2015.2468600.

[7] M. Ali, A. Sahoo, H. I. Nurdin, J. Ravishankar, and J. E. Fletcher, "On the Power Sharing Dynamics of Parallel-Connected Virtual OscillatorControlled and Droop-Controlled Inverters in an AC Microgrid," in
IECON 2019 - 45th Annual Conference of the IEEE Industrial Electronics Society, Lisbon, Portugal, Oct. 2019, pp. 3931-3936. doi: 10.1109/IECON.2019.8927510.

[8] T. Qoria, E. Rokrok, A. Bruyere, B. Francois, and X. Guillaud, "A PLLFree Grid-Forming Control With Decoupled Functionalities for HighPower Transmission System Applications," IEEE Access, vol. 8, pp. 197363-197378, 2020, doi: 10.1109/ACCESS.2020.3034149.

[9] S. D'Arco, J. A. Suul, and O. B. Fosso, "Automatic Tuning of Cascaded Controllers for Power Converters Using Eigenvalue Parametric Sensitivities," IEEE Trans. Ind. Appl., vol. 51, no. 2, pp. 1743-1753, Mar. 2015, doi: 10.1109/TIA.2014.2354732.

[10] Z. Li, C. Zang, P. Zeng, H. Yu, S. Li, and J. Bian, "Control of a GridForming Inverter Based on Sliding-Mode and Mixed $H_{2} / H_{\infty}$ Control," IEEE Trans. Ind. Electron., vol. 64, no. 5, pp. 3862-3872, May 2017, doi: 10.1109/TIE.2016.2636798.

[11] T. Qoria, F. Gruson, F. Colas, X. Guillaud, M. Debry, and T. Prevost, "Tuning of Cascaded Controllers for Robust Grid-Forming Voltage Source Converter," in 2018 Power Systems Computation Conference (PSCC), Jun. 2018, pp. 1-7. doi: 10.23919/PSCC.2018.8443018.

[12] X. Wang, M. G. Taul, H. Wu, Y. Liao, F. Blaabjerg, and L. Harnefors, "Grid-Synchronization Stability of Converter-Based Resources-An Overview," IEEE Open J. Ind. Appl., vol. 1, pp. 115-134, 2020, doi: 10.1109/OJIA.2020.3020392.

[13] K. Yu, Q. Ai, S. Wang, J. Ni, and T. Lv, "Analysis and optimization of droop controller for microgrid system based on small-signal dynamic model," IEEE Trans. Smart Grid, vol. 7 no. 2, pp. 695-705, Mar. 2016.

[14] S. Wang, Z. Liu, J. Liu, D. Boroyevich, and R. Burgos, "Small-signal modeling and stability prediction of parallel droop-controlled inverters based on terminal characteristics of individual inverters," IEEE Trans. Power Electron., Early Access, DOI: 10.1109/TPEL.2019.2914176.

[15] Y. Liao, X. Wang and F. Blaabjerg, "Passivity-Based Analysis and Design of Linear Voltage Controllers For Voltage-Source Converters," in IEEE Open Journal of the Industrial Electronics Society, vol. 1, pp. 114-126, 2020, doi: 10.1109/OJIES.2020.3001406.

[16] T. QORIA, F. GRUSON, F. Colas, X. Kestelyn, and X. Guillaud, "Analysis of the coupling between the outer and inner control loops of a Grid-forming Voltage Source Converter," EPE2020, Lyon, FRANCE, p. 10, Aug. 2020.

[17] Y. Liao, X. Wang, F. Liu, K. Xin and Y. Liu, "Sub-Synchronous Control Interaction in Grid-Forming VSCs with Droop Control," 2019 4th IEEE Workshop on the Electronic Grid (eGRID), 2019, pp. 1-6, doi: 10.1109/eGRID48402.2019.9092640.

[18] R. Rosso, X. Wang, M. Liserre, X. Lu, and S. Engelken, "GridForming Converters: Control Approaches, Grid-Synchronization, and Future Trends-A Review," IEEE Open J. Ind. Appl., vol. 2, pp. 93-109, 2021, doi: 10.1109/OJIA.2021.3074028.

[19] Y. Liao and xiongfei wang, "Impedance Decomposition for DesignOriented Analysis of Grid-Forming Voltage-Source Converters," preprint, Aug. 2020. doi: 10.36227/techrxiv.12855167.v1.

[20] Q. Cossart, F. Colas, and X. Kestelyn, "A Novel Event- and NonProjection-Based Approximation Technique by State Residualization for the Model Order Reduction of Power Systems with a High Renewable Energies Penetration,” IEEE Trans. Power Syst., pp. 1-1, 2020, doi: 10.1109/TPWRS.2020.3010891.

[21] L. Zhang, L. Harnefors, and H. Nee, "Power-Synchronization Control of Grid-Connected Voltage-Source Converters," IEEE Trans. Power Syst., vol. 25, no. 2, pp. 809-820, May 2010, doi: 10.1109/TPWRS.2009.2032231.

[22] T. Qoria, C. Li, K. Oue, F. Gruson, F. Colas, and X. Guillaud, "Direct AC voltage control for grid-forming inverters," J. Power Electron., vol. 20, no. 1, pp. 198-211, Jan. 2020, doi: 10.1007/s43236-019-00015-4.

[23] X. Lu, J. Wang, J. M. Guerrero, and D. Zhao, "Virtual-ImpedanceBased Fault Current Limiters for Inverter Dominated AC Microgrids," IEEE Trans. Smart Grid, vol. 9, no. 3, pp. 1599-1612, May 2018, doi: 10.1109/TSG.2016.2594811.

[24] A. D. Paquette and D. M. Divan, "Virtual Impedance Current Limiting for Inverters in Microgrids With Synchronous Generators," IEEE Trans. Ind. Appl., vol. 51, no. 2, pp. 1630-1638, Mar. 2015, doi: 10.1109/TIA.2014.2345877.

[25] T. Qoria, F. Gruson, F. Colas, G. Denis, T. Prevost, and X. Guillaud, "Critical clearing time determination and enhancement of gridforming converters embedding virtual impedance as current limitation algorithm," IEEE J. Emerg. Sel. Top. Power Electron., pp. 1-1, 2019, doi: 10.1109/JESTPE.2019.2959085.

[26] P. Aristidou. "Time-domain simulation of large electric power systems using domain decomposition and parallel processing methods," Dissertation, Université de Liege, June 2015. 